\title{
Extracción purificación y caracterización de inhibidores de tripsina provenientes de semillas andinas
}

\author{
(Extraction purification and characterization of trypsin \\ inhibitors from Andean seeds)
}

Patricio Castillo ${ }^{1}$, Lorena Quinchuela ${ }^{1}$, Paulina Echeverría ${ }^{1}$, Gonzalo Jácome ${ }^{1}$

\begin{abstract}
Resumen:
El presente trabajo determinó las mejores condiciones para inmovilizar covalentemente tripsina en una matriz de sepharosa, con el fin de aplicarla en la purificación de inhibidores de la misma proteasa. Los mayores valores de retención de actividad funcional y de actividad enzimática inmovilizada se obtuvieron en una matriz de Sepharosa 6B-CL a temperatura ambiente, a un $\mathrm{pH}$ de 10,5, con una carga enzimática de $25 \mathrm{mg} / \mathrm{mL}$ de matriz, en un tiempo mínimo de inmovilización de $12 \mathrm{~h}$, para alcanzar un preparado inmovilizado estable. Posteriormente, se seleccionaron los inhibidores de tripsina más activos a través de la comparación de extractos provenientes de semillas de amaranto (Amaranthus caudatus L.), arveja (Pisum sativum), lupino o "chocho" (Lupinus mutabilis), fréjol (Phaseolus vulgaris) y sangorache (Amaranthus hybridus $L$.) que fueron purificados parcialmente mediante ultrafiltración centrífuga, tratamiento calórico y precipitación con TCA. El permeado y retenido de sangorache fueron los extractos inhibidores más activos y se purificaron selectivamente, por separado, mediante cromatografía de afinidad en la matriz de tripsina-glioxil-sepharosa 6B-CL. Su caracterización cinética determinó la presencia de dos inhibidores; el inhibidor I que correspondió a una inhibición competitiva y el inhibidor II, a una de tipo mixta.
\end{abstract}

Palabras clave: inhibidores de tripsina; inmovilización covalente; Amaranthus hybridus $L$.

\begin{abstract}
:
This work established the conditions of covalent immobilization of trypsin on a Sepharose matrix, which could be applied for the purification of trypsin inhibitors. The higher values of retention of enzymatic activity and immobilized enzymatic activity were obtained with a Sepharose 6B-CL matrix, at room temperature, a pH value of 10.5 , an enzymatic load of 25 $\mathrm{mg} / \mathrm{mL}$, and a minimum immobilization time of 12 hours, in order to obtain a stable immobilization. The most active trypsin inhibitors were selected through the comparison of, extracts obtained from the seeds of amaranth (Amaranthus caudatus L.), pea (Pisum sativum), lupine or "chocho" (Lupinus mutabilis), bean (Phaseolus vulgaris) and "sangorache" (Amaranthus hybridus L.). The inhibitors were partially purified using centrifugal ultrafiltration, heat treatment, and TCA precipitation. The permeated and retained fractions of "sangorache" were selected as the most active trypsin inhibitors, and they were selectively purified using affinity chromatography in a Trypsin - Glyoxyl - Sepharose 6B-CL matrix. The kinetic characterization showed the presence of two inhibitors; the first one corresponded to a competitive inhibitor, while the second one behaved as a mixed inhibitor.
\end{abstract}

Keywords: Trypsin inhibitor; covalent immobilization; Amaranthus hybridus L.

\footnotetext{
${ }^{1}$ Escuela Politécnica Nacional, Quito - Ecuador ( \{patricio.castillo, lorena.quinchuelal, paulina.echeverria, gonzalo.jacome\} @epn.edu.ec )
} 


\section{Introducción}

Los inhibidores de proteasas (IPs) son proteínas de bajo peso molecular, comunes en el reino vegetal (Habib y Majid, 2007), donde actúan como parte de los mecanismos de defensa de las plantas, ya que inhiben la actividad de las proteasas digestivas como tripsina y quimotripsina, de insectos y otros organismos pequeños, de forma que provocan la pérdida de aminoácidos esenciales, que altera su normal crecimiento (Elizalde, Portilla y Chaparro, 2009).

La obtención y purificación de IPs tienen un alto potencial en varios campos, en especial en la agricultura, puesto que su aplicación como bioinsecticidas específicos puede remplazar a productos sintéticos nocivos para el ambiente (García et al., 2009; Habib y Majid, 2007).

La tripsina es una proteasa que posee una triada catalítica en su centro activo, constituida por residuos de serina, histidina y ácido aspártico, lo que permite la hidrólisis específica de péptidos o proteínas con residuos de lisina y arginina (Müller, 2008). Su uso tiene algunas ventajas a escala industrial, pero se ha visto restringida debido a su termolabilidad, solubilidad en el medio de reacción y pérdida de actividad proteolítica (Tripathi, 2009).

Con el fin de extender sus aplicaciones, la inmovilización covalente, a través de la unión entre la enzima y un soporte capaz de reaccionar con ella, ha permitido reducir las limitaciones que pueden ser causadas por condiciones de operación agresivas, como valores de $\mathrm{pH}$ lejanos al óptimo, agitación excesiva o elevadas presiones (Koolman y Röhm, 2004). Adicionalmente, el proceso de inmovilización permite obtener un preparado capaz de ser empleado en la purificación de inhibidores de proteasas, mediante cromatografía de afinidad (Del Monte et al., 2014).

La purificación de los IP puede ser complicada, usualmente se encuentran dentro de las hojas, el tallo o las raíces de las plantas aunque están en mayor proporción en las semillas; por lo cual, son necesarios protocolos que permitan obtener de formas más puras estos elementos. Dentro de las técnicas de mayor selectividad se encuentra la cromatografía de afinidad ya que por su alta eficiencia permite obtener a los inhibidores con elevada pureza; esta técnica aprovecha la afinidad biológica reversible entre los inhibidores y las enzimas sobre las que actúan. Este protocolo es ampliamente usado por su corto tiempo de operación, alta capacidad de selección y porque permite procesar volúmenes considerables de muestra (Dos Santos et al., 2012).

Debido a que algunos estudios señalan que las semillas de cereales y leguminosas andinas presentan altos contenidos de inhibidores de tripsina (Elizalde, Portilla y Chaparro, 2009; Ramírez y Rangel, 2011), en la presente investigación se planteó obtener y caracterizar extractos inhibidores de tripsina purificados por cromatografía de afinidad, en una matriz de tripsina-glioxilsepharosa a partir de semillas de amaranto (Amaranthus caudatus L.), arveja (Pisum sativum), chocho (Lupinus mutabilis), fréjol (Phaseolus vulgaris) y sangorache (Amaranthus hybridus L.), que pueden ser utilizados en la formulación de potenciales bioinsecticidas. 


\section{Metodología}

\subsection{Materiales}

Las semillas de amaranto (Amaranthus caudatus L.), arveja (Pisum sativum, var. I-432-Lojanita), chocho (Lupinus mutabilis, var. I-450-Andino), fréjol (Phaseolus vulgaris, var. I-414-Yunguilla) y sangorache (Amaranthus hybridus L.), fueron obtenidas del Instituto Nacional Autónomo de Investigaciones Agropecuarias (INIAP). Se empleó tripsina en polvo de marca GIBCO; el sustrato enzimático BApNA, la sepharosa 4B-CL y 6B-CL y glicidol fueron marca SIGMA. Todos los reactivos empleados superan el $98 \%$ de pureza.

\subsection{Preparación de la matriz de afinidad}

\subsubsection{Activación de la sepharosa}

Se activaron $75 \mathrm{~g}$ de sepharosa en medio básico y reductor, con $35 \mathrm{~mL}$ de hidróxido de sodio 1,7 M y 1,016 g de borohidruro de sodio. Con el fin de obtener gliceril-sepharosa, se agregaron 25 $\mathrm{mL}$ de glicidol y se mantuvo en agitación constante durante $16 \mathrm{~h}$. Al cabo de este tiempo, se filtró y lavó con agua destilada la gliceril-sepharosa obtenida. Posteriormente, se añadió una solución de peryodato de sodio $0,12 \mathrm{M}$ y se agitó la mezcla durante $1 \mathrm{~h}$ para conseguir glioxil-sepharosa. Finalmente, se realizaron seis lavados con agua destilada, que fueron recolectados para la consecuente determinación del grado de oxidación de la sepharosa (Freije et al., 2005). La cantidad de peryodato de sodio presente en las fracciones obtenidas en los lavados de la sepharosa se determinó de acuerdo con la técnica descrita por Bonzón (1996).

\subsubsection{Selección del tamaño de poro en el proceso de inmovilización covalente}

La selección de cada uno de los parámetros de inmovilización se realizó con base en tres variables de respuesta: el porcentaje de retención de actividad funcional (\%RAF), el porcentaje de enzima inmovilizada \%El y el porcentaje de actividad enzimática inmovilizada \%AEI. Estos parámetros se calcularon de acuerdo con las ecuaciones descritas por Li, Gao, Wei, Qu, Ma y Zhou (2010).

Para seleccionar el tamaño de poro de la sepharosa, se colocaron $10 \mathrm{~mL}$ de solución enzimática, equivalentes a $28 \mathrm{mg}$ de tripsina/mL de sepharosa, con $3 \mathrm{~mL}$ de glioxil-sepharosa a $\mathrm{pH} 10$ y temperatura ambiente $\left(15^{\circ} \mathrm{C}\right)$. Se emplearon dos tamaños de poro, correspondientes a la sepharosa 4 B-CL y 6 B-CL, previamente activadas. Se mantuvo el contacto entre la enzima y el soporte durante 24 h. Posteriormente, se filtró y lavó el preparado inmovilizado. Se redujo la tripsina inmovilizada con borohidruro de sodio, durante $30 \mathrm{~min}$, en baño de hielo para finalmente filtrar y lavar tres veces el preparado con soluciones amortiguadoras de Tris $\mathrm{HCl} 0,1 \mathrm{M} \mathrm{pH}$, acetato 0,1 M pH 4 y borato 0,1 M, pH 10 (Bonzón, 1996; Del Monte et al., 2014). 


\subsubsection{Definición de la temperatura en el proceso de inmovilización covalente}

Se inmovilizaron durante 24 h, $10 \mathrm{~mL}$ de solución enzimática de $28 \mathrm{mg} / \mathrm{mL}$ de matriz y $\mathrm{pH} 10$, con $3 \mathrm{~mL}$ del soporte de tamaño de poro previamente seleccionado, a dos temperaturas, de 15 y $35 \stackrel{\circ}{\circ}$. Los procesos de reducción y lavado del preparado inmovilizado se realizaron de la manera descrita en la sección anterior.

\subsubsection{Influencia de la carga enzimática y del pH en el proceso de inmovilización covalente}

El efecto de las combinaciones de $\mathrm{pH}$ y carga enzimática se definieron mediante un diseño experimental de composición central con dos factores; los rangos de experimentación fueron para el $\mathrm{pH}$ de 8 a 12 y para la carga enzimática de 25 a $85 \mathrm{mg}$ tripsina/mL sepharosa (Montgomery, 2003).

La temperatura y tipo de sepharosa empleados fueron los determinados mediante los ensayos anteriores. Además, el tiempo de inmovilización, el proceso de reducción y los lavados del preparado se ejecutaron de acuerdo con la sección 2.2.2.

\subsubsection{Determinación del mejor proceso de inmovilización covalente}

Se inmovilizó tripsina por $24 \mathrm{~h}$ a las condiciones de tamaño de poro, $\mathrm{pH}$, temperatura y carga enzimática determinadas en las secciones anteriores. Durante este tiempo, se tomaron alícuotas del sobrenadante cada $\mathrm{h}$ y se midieron sus actividades proteolíticas. Se ajustaron los datos a una regresión de decaimiento exponencial y se estableció el mínimo tiempo de inmovilización, que correspondió al tiempo en el que alcanza la insolubilización y la estabilización de la interacción covalente.

\subsection{Aplicación de la matriz tripsina glioxil-sepharosa en la purificación de inhibidores enzimáticos}

\subsubsection{Obtención de extractos clarificados}

Las semillas fueron molidas hasta obtener una harina fina, que posteriormente fue desengrasada mediante extracciones sólido-líquido con 1-propanol, en una relación peso:volumen 1:4, en intervalos de $30 \mathrm{~min}$. La extracción se repitió hasta que el filtrado no presentó turbidez. La harina desengrasada fue secada a $30^{\circ} \mathrm{C}$ por $16 \mathrm{~h}$ (Betancur, Guerrero, Hernández y Marrufo, 2012).

Los extractos crudos se prepararon con la suspensión de la harina seca en tampón fosfato de sodio $50 \mathrm{mM}$, con una relación 1:5 durante $1,5 \mathrm{~h}$, con agitación constante. Se eliminó el material insoluble mediante centrifugación a $835 \times \mathrm{g}$ durante $30 \mathrm{~min}$. El extracto fue fraccionado por ultrafiltración centrífuga, en primer lugar con una membrana Millipore con un tamaño de poro de $50 \mathrm{kDa}$, durante $40 \mathrm{~min}$ a $2000 \times \mathrm{g}$; finalmente, con una membrana Millipore con un tamaño de 
poro de $10 \mathrm{kDa}$, durante $40 \mathrm{~min}$ a $1163 \times \mathrm{g}$. Se denominó, retenido a las fracciones superiores a $10 \mathrm{kDa}$ y permeado a aquellas con pesos moleculares inferiores a este valor (Chávez et al., 2011).

Los permeados y retenidos de cada semilla fueron purificados mediante un tratamiento combinado; se empleó en primer lugar, un tratamiento calórico, en el cual se sometió a los extractos a calentamiento en baño María, durante $30 \mathrm{~min}$ a $60{ }^{\circ} \mathrm{C}$; seguido de un proceso de precipitación de proteínas con ácido tricloro acético (TCA), en una relación 1:5. El TCA fue removido de las muestras mediante filtración en gel en una columna PD-10, Sephadex G-25M, Pharmacia (Chávez et al., 2011).

Para caracterizar a los extractos antes y después de la purificación se determinaron la actividad enzimática, actividad inhibidora, concentración de proteína y actividad inhibidora específica. La actividad de tripsina se determinó por el método de Earlanger et al. (1961) con la hidrólisis del sustrato BApNA seguida a 400 nm (Castillo, Gómez, Sinche, Duchicela y Muñoz, R., 2012). La actividad inhibidora ( $\mathrm{Al}$ ) se determinó como la diferencia en la actividad enzimática de tripsina en presencia y ausencia del inhibidor. La concentración de proteína ([P]) se estableció con el método de absorción en el ultravioleta a $280 \mathrm{~nm}$ (Aitken y Learmoth, 2002). La actividad inhibidora específica (AIE) se calculó como la relación entre la Al y [P] de cada muestra; este parámetro se consideró para elegir a los inhibidores de tripsina más activos en los permeados y retenidos.

\subsubsection{Purificación de los inhibidores de tripsina más activos mediante cromatografía de afinidad}

Se preparó la matriz de afinidad tripsina-glioxil-sepharosa 6B-CL y se empacó este gel en una columna del equipo para cromatografía; se lavó el sistema con solución tampón fosfato de sodio

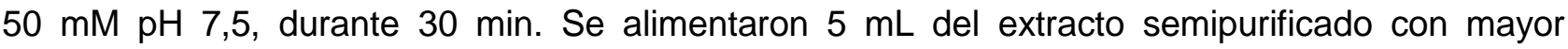
actividad de tripsina. Los inhibidores fueron eluidos con una solución de $\mathrm{KCl}-\mathrm{HCl} 1 \mathrm{M} \mathrm{pH}$ 1,9. Se recogieron fracciones de $3 \mathrm{~mL}$, de forma continua. Los picos de fracciones con $\mathrm{Al}$ y $[\mathrm{P}]$ fueron agrupados en lotes que fueron liofilizados a una temperatura de $67^{\circ} \mathrm{C}$ y un vacío de $10 \mathrm{~Pa}$.

\subsubsection{Caracterización de los inhibidores de tripsina más activos}

Se realizó la caracterización cinética con la determinación de la constante de Michaelis-Menten $(\mathrm{Km})$, velocidad máxima (Vmáx) y constante de inhibición (Ki).

Con el fin de determinar estos parámetros, se midió la actividad enzimática de tripsina en ausencia de inhibidor y en presencia de iguales volúmenes de alícuotas de los picos A, B, C, D, E y $F$, frente a soluciones del sustrato BApNA en concentraciones 0,$58 ; 0,96 ; 1,34 ; 2,11 ; 2,87 \mathrm{mM}$. A través del método de Lineweaver-Burk, se determinaron Km y Vmáx. Finalmente, con el análisis de estos dos parámetros se determinó la Ki (Nelson y Cox, 2014). 


\subsection{Análisis estadístico}

El análisis estadístico de los resultados correspondientes a \%El, \%AEI, \%RAF, AI, [P] y AIE fue realizado mediante un ANOVA y la prueba de rangos múltiples por el método de Fisher, con un nivel de confianza del $95 \%$, con el uso del paquete estadístico Statgraphics Centurion XVI.

\section{Resultados y Discusión}

\subsection{Preparación de la matriz de afinidad}

\subsubsection{Selección del tamaño de poro en el proceso de inmovilización covalente}

Antes de inmovilizar la tripsina, al activar la sepharosa 4B-CL y 6 B-CL y se obtuvieron los grados de oxidación (GOM) de 216,66 \pm 7,30 y 220,59 \pm 9,71 $\mu \mathrm{mol}$ NalO4/mL matriz, respectivamente. Se realizó un análisis de varianza para estos resultados y se encontró que no existe diferencia estadísticamente significativa entre los GOM, por lo tanto el tamaño de poro no influye en el proceso de activación; por lo que, se presume que se formó aproximadamente el mismo número de grupos carbonilo en el soporte. Para seleccionar el tipo de sepharosa se emplearon los dos soportes activos para inmovilizar la tripsina y se encontraron las variables de respuesta que se presentan en la Tabla 1.

Tabla 1. \%RAF, \%El y \%AEI para tripsina inmovilizada en sepharosa 4 B-CL y 6 B-CL

\begin{tabular}{|c|c|c|}
\hline $\begin{array}{c}\text { Variable de } \\
\text { respuesta }\end{array}$ & $\begin{array}{c}\text { Sepharosa } \\
\text { 4B-CL }\end{array}$ & $\begin{array}{c}\text { Sepharosa } \\
\text { 6B-CL }\end{array}$ \\
\hline$\%$ RAF & $25,78 \pm 0,68^{\mathrm{A}}$ & $33,03 \pm 1,31^{\mathrm{B}}$ \\
\hline$\% \mathrm{EI}$ & $89,28 \pm 1,28^{\mathrm{C}}$ & $91,20 \pm 2,33^{\mathrm{C}}$ \\
\hline$\%$ AEI & $87,68 \pm 3,49^{\mathrm{D}}$ & $96,43 \pm 0,54^{\mathrm{E}}$ \\
\hline
\end{tabular}

$\bar{X} \pm \sigma(n=3)$

Letras diferentes en la misma fila indican diferencias estadísticamente significativas (Fisher, $95 \%$ de confianza)

Los mayores valores de \%RAF y \%AEI se obtuvieron con sepharosa 6B-CL. Asimismo, los \%EI fueron iguales para los dos niveles de tamaño de poro. Esto indica que el preparado inmovilizado en sepharosa 6B-CL presenta mayor actividad proteolítica; probablemente porque la tripsina se fijó en la superficie fuera de los poros de la matriz, lo que pudo disminuir las dificultades difusionales, que podría provocar el proceso de inmovilización (Da Silva et al., 2012). Al obtener mayores valores de las variables de respuesta, se decidió trabajar con sepharosa 6B-CL en los ensayos posteriores.

\subsubsection{Definición de la temperatura en el proceso de inmovilización covalente}

Los valores obtenidos de las variables de respuesta, luego de la inmovilización en sepharosa 6B$\mathrm{CL}$ a 15 y $35^{\circ} \mathrm{C}$, se muestran en la Tabla 2. 
Tabla 2. \%RAF, \%El y \%AEI para tripsina inmovilizada a 15 y $35^{\circ} \mathrm{C}$

\begin{tabular}{|c|c|c|}
\hline \multirow{2}{*}{$\begin{array}{c}\text { Variable de } \\
\text { Respuesta }\end{array}$} & $\mathbf{3 5}^{\circ} \mathbf{C}$ & $\mathbf{1 5}^{\circ} \mathbf{C}$ \\
\cline { 2 - 3 } \%RAF & $22,29 \pm 1,20^{\mathrm{A}}$ & $33,03 \pm 1,31^{\mathrm{B}}$ \\
\hline \%EI & $87,79 \pm 1,09^{\mathrm{C}}$ & $91,20 \pm 2,33^{\mathrm{C}}$ \\
\hline \%AEI & $98,72 \pm 0,17^{\mathrm{D}}$ & $96,43 \pm 0,54^{\mathrm{E}}$ \\
\hline
\end{tabular}

Se encontró diferencia significativa entre los valores de \%RAF y \%AEI al inmovilizar la enzima a 15 y $35^{\circ} \mathrm{C}$. Se obtuvo un \%RAF mayor al emplear la temperatura de $15^{\circ} \mathrm{C}$, en tanto que ocurrió lo contrario con el \%AEI. Debido a que el \%RAF toma en cuenta la actividad del preparado inmovilizado y mostró mayor valor a $15^{\circ} \mathrm{C}$, se escogió esta temperatura para buscar la mejor carga enzimática y el pH de trabajo.

\subsubsection{Influencia de la carga enzimática y del pH en el proceso de inmovilización covalente}

En la Tabla 3, los parámetros \%RAF, \%AEI, \%El para las combinaciones pH y carga enzimática planteados por el diseño de composición central con dos factores.

Tabla 3. Parámetros \%RAF, \%AEI y \%El a diferente carga enzimática y valores de $\mathrm{pH}$

\begin{tabular}{|c|c|c|c|c|}
\hline $\begin{array}{c}\text { Carga } \\
\mathbf{( m g} \mathbf{m L})\end{array}$ & $\mathbf{p H}$ & $\%$ RAF & \%AEI & \%EI \\
\hline 25,0 & 10,0 & 35,17 & 95,26 & 88,94 \\
\hline 33,7 & 8,6 & 17,51 & 59,56 & 91,29 \\
\hline 33,7 & 11,4 & 24,83 & 97,47 & 95,27 \\
\hline 55,0 & 8,0 & 19,74 & 64,32 & 85,48 \\
\hline 55,0 & 10,0 & 23,73 & 91,64 & 89,03 \\
\hline 55,0 & 10,0 & 23,27 & 93,19 & 88,97 \\
\hline 55,0 & 10,0 & 22,85 & 93,60 & 89,09 \\
\hline 55,0 & 12,0 & 22,72 & 98,60 & 91,90 \\
\hline 76,3 & 8,6 & 11,94 & 43,12 & 90,39 \\
\hline 76,3 & 11,4 & 24,42 & 95,69 & 89,38 \\
\hline 85,0 & 10,0 & 12,03 & 67,56 & 87,69 \\
\hline
\end{tabular}

Según los valores tabulados, el mayor \%RAF fue 35,17, que se obtuvo a $\mathrm{pH} 10$ y carga de $25 \mathrm{mg} / \mathrm{mL}$ de matriz. El análisis de varianza evidenció que la carga enzimática influye significativamente sobre la variable de respuesta (\%RAF). También se encontró que la actividad proteolítica directa no presenta diferencias proporcionales con relación al incremento de la carga, es así que para las cargas 25,0; 55,0 y $85,0 \mathrm{mg} / \mathrm{mL}$ se obtuvieron valores de actividad directa de 4,$71 ; 6,43$ y $6,03 \mathrm{mU} / \mathrm{mL}$ de matriz, respectivamente.

Como se observa en la Tabla 3, los mayores valores de \%AEI se obtienen al trabajar en medio más alcalino. El análisis estadístico determinó que el único factor que presentó diferencia significativa, a un nivel de confianza del 95\%, fue el pH. Así también la Tabla 3 indica los 
valores de \%El los cuales mediante el análisis estadístico muestran que no existen diferencias significativas con la variación del pH y carga enzimática.

El máximo valor de \%RAF estimado mediante la opción estadística "Optimizar Respuesta" que ofrece el Stagraphics Centurion XVI se encuentra en la Tabla 4.

Tabla 4. Valores de $\mathrm{pH}$ y carga enzimática determinados para maximizar el \%RAF

\begin{tabular}{|c|c|c|c|}
\hline \multirow{2}{*}{ Factor } & \multicolumn{3}{|c|}{ VALOR } \\
\cline { 2 - 4 } & Bajo & Alto & Calculado \\
\hline $\mathrm{pH}$ & 8,00 & 12,00 & 10,50 \\
\hline $\begin{array}{c}\text { Carga } \\
(\mathrm{mg} / \mathrm{mL})\end{array}$ & 25,00 & 85,00 & 25,00 \\
\hline \multicolumn{2}{|c|}{ Valor máximo para \%RAF } & 29,27 \\
\hline
\end{tabular}

Los valores resultantes de la optimización para obtener el máximo \%RAF son $25 \mathrm{mg} / \mathrm{mL}$ de enzima inmovilizada a pH 10,5. El medio básico es necesario para la unión covalente entre los grupos amino de la enzima y los grupos aldehído del soporte. Sin embargo, cuando se sobrepasa el límite de pH predicho por el modelo, es probable que predomine el fenómeno de desnaturalización de la tripsina, que produciría pérdida de la actividad directa.

\subsubsection{Determinación del mejor proceso de inmovilización covalente}

La Figura 1 muestra el decaimiento de la actividad enzimática en el sobrenadante durante el tiempo de inmovilización.

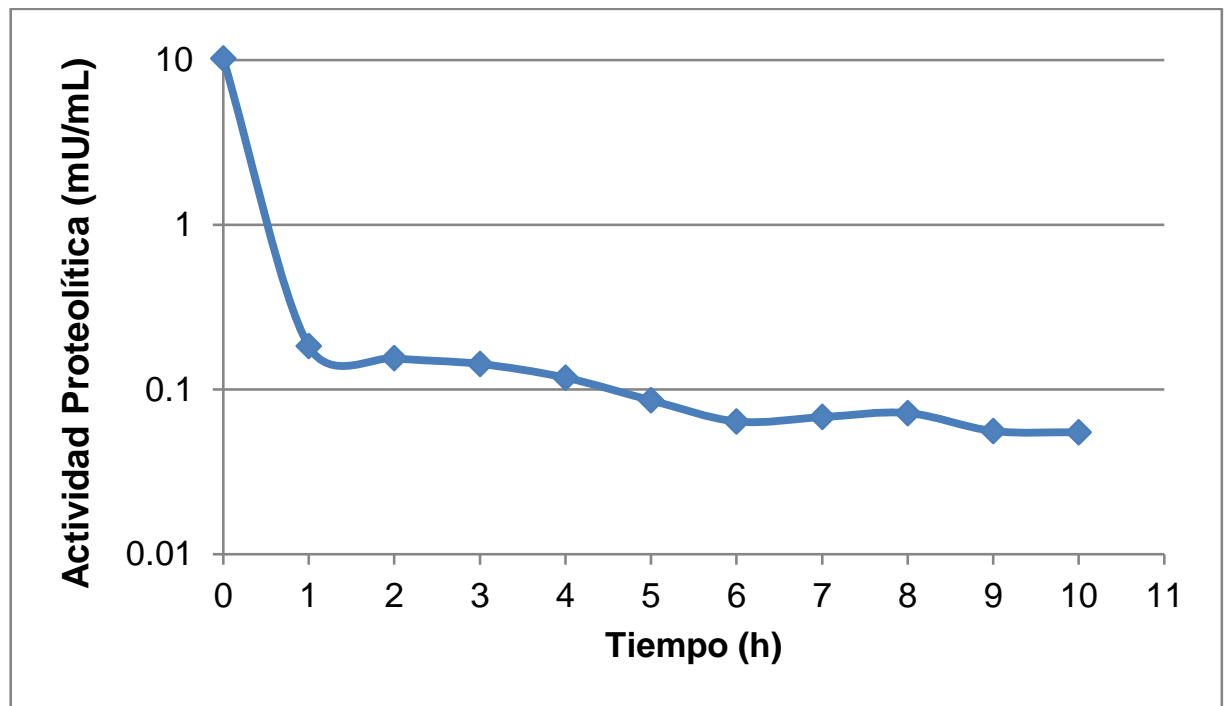

Figura 1. Correlación de decaimiento entre la actividad proteolítica del sobrenadante y el tiempo de inmovilización

La Figura 1 señala que el mínimo tiempo de contacto entre la enzima y el soporte debería ser $1 \mathrm{~h}$, aunque Rocha, Goncalves y Teixeira, (2011) afirman que durante este período se produciría la insolubilización de la tripsina en el soporte pero la fase de interacción covalente requiere 
tiempos mayores a 10 horas; la razón de este comportamiento radica en que durante esta segunda fase ocurre el alineamiento entre los grupos amino de la tripsina y los carbonilo aldehídico del soporte (Da Silva et al., 2012). Con base en este criterio se decidió fijar un tiempo mínimo de inmovilización igual a 12 horas.

La purificación de inhibidores de tripsina procedentes de las semillas de amaranto, arveja, chocho, fréjol y sangorache utilizó ultrafiltración centrifuga, tratamiento calórico, precipitación de proteínas con TCA y cromatografía de afinidad en la matriz de afinidad tripsina-glioxil-sepharosa preparada en las mejores condiciones establecidas en esta sección. Los extractos crudos de los inhibidores se realizaron a partir de la suspensión de la harina desengrasada de las semillas en tampón fosfato de sodio.

\subsection{Aplicación de la matriz en la purificación de inhibidores enzimáticos}

\subsubsection{Selección de los inhibidores de tripsina más activos en los extractos clarificados}

Los extractos crudos de cada semilla se fraccionaron en permeados y retenidos mediante ultrafiltración centrífuga, con una membrada de $10 \mathrm{kDa}$ y se purificaron parcialmente por medio de un tratamiento calórico $\left(\mathrm{T}=60^{\circ} \mathrm{C}\right)$ combinado con precipitación de proteínas con TCA $(5 \%)$.

En la Tabla 5 se muestran los resultados de la actividad inhibidora específica (AIE) alcanzado luego del proceso de purificación parcial, también se muestra el análisis estadístico realizado por la prueba de rangos múltiples con un nivel de confianza del 95\%, para tres repeticiones con cada semilla.

Tabla 5. AEI de los permeados y retenidos purificados con tratamiento calórico y precipitación con TCA

\begin{tabular}{|c|c|c|}
\hline \multirow{2}{*}{ Semilla } & \multicolumn{2}{|c|}{ AIE $(\mathrm{mU} / \mathrm{mg})$} \\
\hline & Permeados & Retenidos \\
\hline Amaranto & $17,74 \pm 0,91^{\mathrm{C}}$ & $31,47 \pm 1,55^{\mathrm{AB}}$ \\
\hline Arveja & $14,33 \pm 1,42^{\mathrm{B}}$ & $36,73 \pm 1,81^{\mathrm{C}}$ \\
\hline Chocho & $9,01 \pm 0,58^{A}$ & $30,40 \pm 0,88^{A}$ \\
\hline Fréjol & $14,77 \pm 0,45^{\mathrm{B}}$ & $33,66 \pm 2,14^{\mathrm{B}}$ \\
\hline Sangorache & $19,22 \pm 0,40^{C}$ & $39,88 \pm 0,69^{D}$ \\
\hline
\end{tabular}

La purificación se evidenció con el incremento de la actividad inhibidora específica, como consecuencia de un incremento de la actividad inhibidora y una disminución de la concentración de proteína en todos los extractos, que se podrían atribuir a la eliminación de proteínas que no aportan a la actividad inhibidora.

Sobre la base del valor de actividad inhibidora específica alcanzado, se seleccionaron al permeado y al retenido semipurificados del extracto de sangorache, con valores de actividad de 
$19,22 \pm 0,40$ y $39,88 \pm 0,69 \mathrm{mU} / \mathrm{mg}$ respectivamente, para siguiente etapa de purificación mediante cromatografía de afinidad.

\subsubsection{Purificación de los inhibidores de tripsina más activos}

La cromatografía se desarrolló con una matriz de afinidad tripsina-glioxil-sepharosa 6B-CL, obtenida con un \%El de 88,95, un \%AEl de 95,26 y un \%RAF de 35,7. Se empleó el mismo procedimiento para el permeado y retenido de la semilla de sangorache.

En la Figura 2, se muestra el cromatograma obtenido en la purificación del permeado de sangorache.

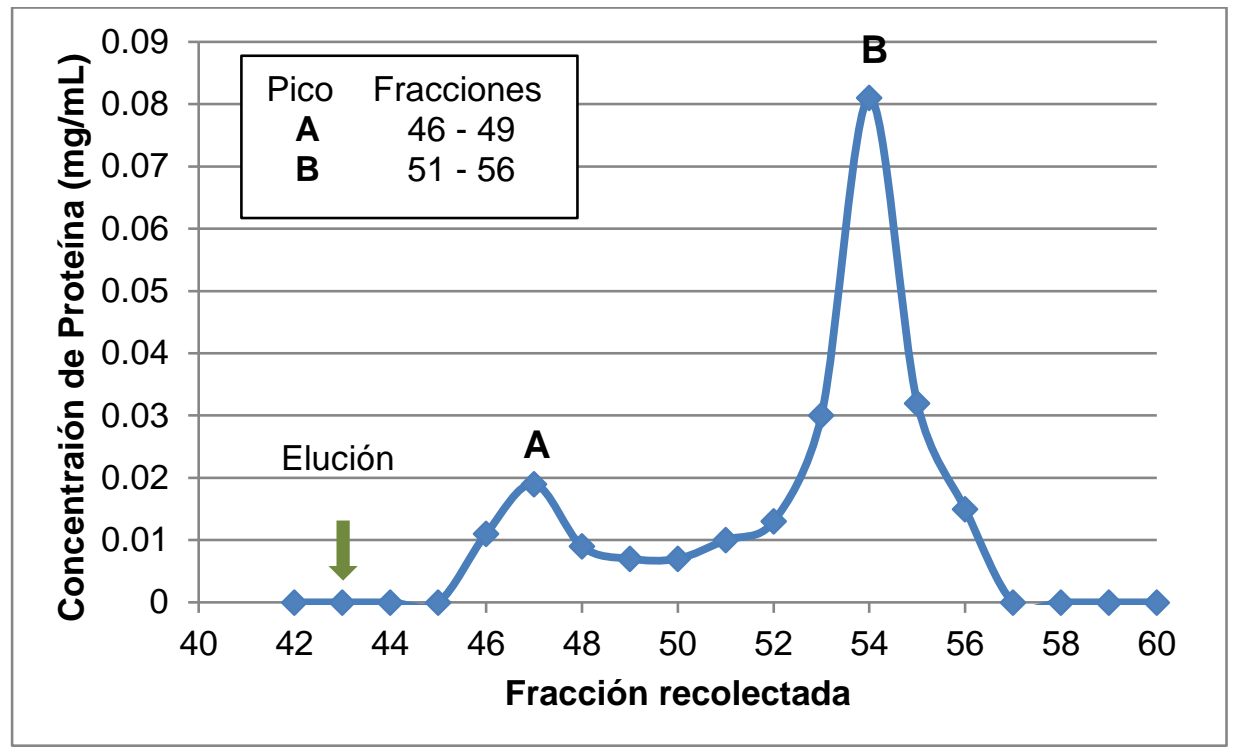

Figura 2. Cromatograma del permeado de sangorache purificado.

Como se observa, después de la elución, se formaron dos picos A y B. Con las fracciones correspondientes se formó un lote con cada pico para determinar los parámetros de control. En la Tabla 6, se indican los resultados de la concentración de proteína, actividad inhibidora y actividad inhibidora específica de los lotes A y B.

Tabla 6. Actividad inhibidora, concentración de proteína y actividad inhibidora específica de los lotes obtenidos del permeado $<10 \mathrm{kDa}$

\begin{tabular}{|c|c|c|c|c|}
\hline \multicolumn{5}{|c|}{ Permeado } \\
\hline Lote & $\begin{array}{c}\text { Volumen } \\
(\mathbf{m L})\end{array}$ & $\begin{array}{c}{[\mathbf{P}]} \\
(\mathbf{m g} / \mathbf{m L})\end{array}$ & $\begin{array}{c}\text { Al } \\
(\mathbf{m U} / \mathbf{m L})\end{array}$ & $\begin{array}{c}\text { AIE } \\
(\mathbf{m U} / \mathbf{m g})\end{array}$ \\
\hline A & 12 & 0,012 & 12,82 & 1114,6 \\
\hline B & 18 & 0,030 & 9,07 & 300,6 \\
\hline
\end{tabular}

Como se observa en la Tabla 6, los lotes A y B, que corresponden a las proteínas que se retuvieron en la matriz, muestran diferentes actividades inhibidoras específicas de tripsina, que podrían estar relacionadas con la existencia de dos inhibidores en el permeado de sangorache. 
Se repitió el procedimiento de purificación para el retenido de la semilla de sangorache y se obtuvo el cromatograma que se observa en la Figura 3.

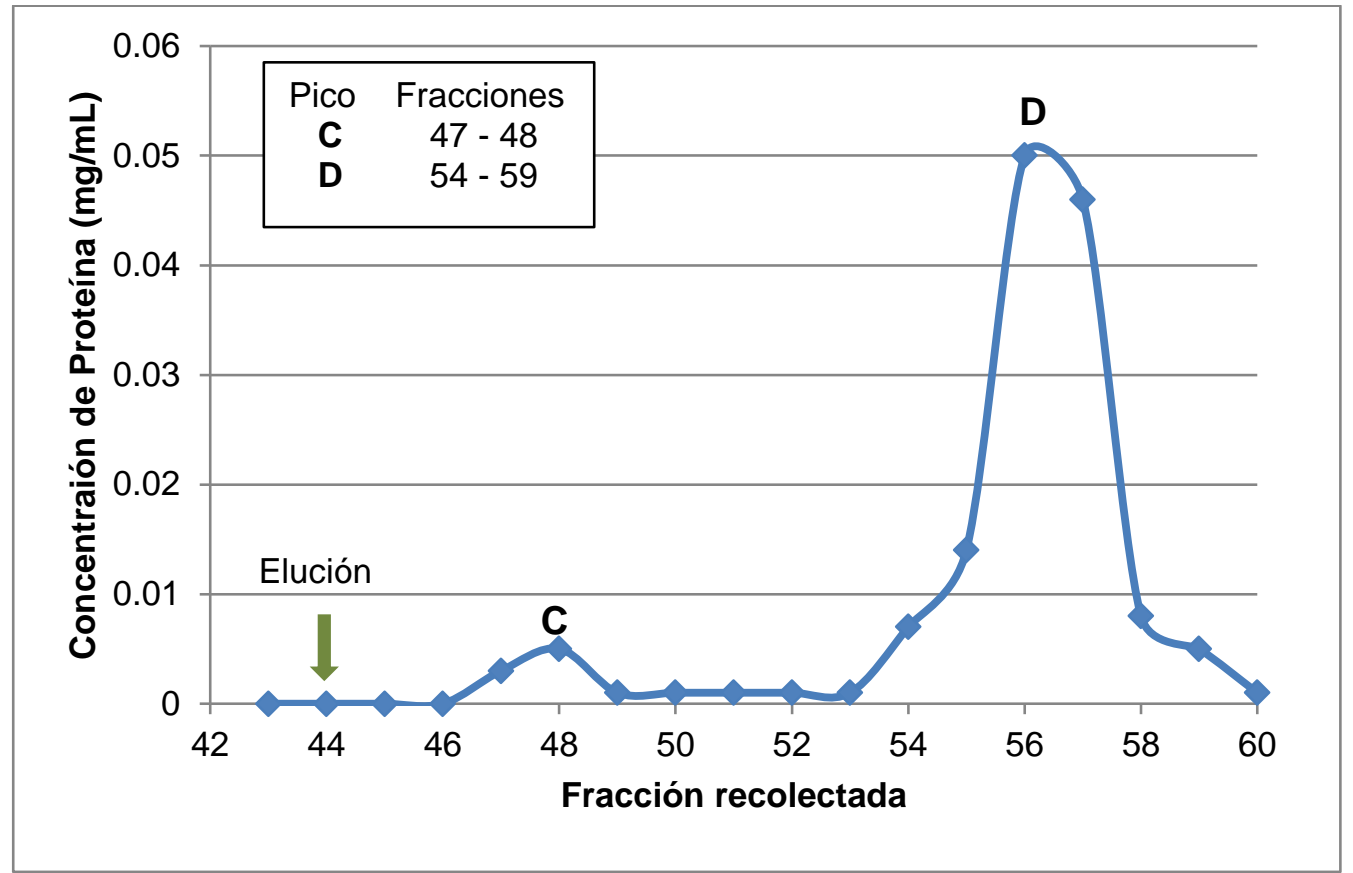

Figura 3. Cromatograma del retenido de sangorache purificado

De igual forma que en el permeado, luego de la elución se formaron 2 picos $\mathrm{C}$ y $\mathrm{D}$. Con las fracciones correspondientes se formó un lote con cada pico para determinar los parámetros de control. En la Tabla 7, se indican los resultados de la concentración de proteína, actividad inhibidora y actividad inhibidora específica de los lotes $C$ y D.

Tabla 7. Actividad inhibidora, concentración de proteína y actividad inhibidora específica de los lotes obtenidos del retenido $>10 \mathrm{kDa}$

\begin{tabular}{|c|c|c|c|c|}
\hline \multicolumn{5}{c|}{ Retenido } \\
\hline Lote & $\begin{array}{c}\text { Volumen } \\
(\mathbf{m L})\end{array}$ & $\begin{array}{c}{[\mathrm{P}]} \\
(\mathbf{m g} / \mathbf{m L})\end{array}$ & $\begin{array}{c}\text { Al } \\
(\mathbf{m U} / \mathbf{m L})\end{array}$ & $\begin{array}{c}\text { AIE } \\
(\mathbf{m U} / \mathbf{m g})\end{array}$ \\
\hline C & 6 & 0,004 & 13,15 & 3288,33 \\
\hline D & 18 & 0,022 & 9,99 & 461,07 \\
\hline
\end{tabular}

\subsubsection{Caracterización de los inhibidores de tripsina más activos}

En la Tabla 8, se muestran los parámetros cinéticos de Vmáx y $\mathrm{Km}$ determinados en ausencia de inhibidor y con alícuotas de los lotes $A, B, C$ y D, mediante el método de linealización de Lineweaver-Burk.

Sobre la base del análisis de los parámetros cinéticos Vmáx y $\mathrm{Km}$, se podría determinar la presencia de dos inhibidores en el extracto de sangorache. El Inhibidor I, en el Lote A y el Inhibidor II, en el lote B del permeado y los lotes C y D del retenido. 
Tabla 8. Parámetros cinéticos determinados sin inhibidor y en presencia de los lotes A, B, C y D

\begin{tabular}{|c|c|c|}
\hline & \multicolumn{2}{|c|}{ Parámetro } \\
\hline & Vmáx (U/mL) & $\mathrm{Km}(\mathrm{mM})$ \\
\hline Sin inhibidor & $4,73 \pm 0,27^{A}$ & $11,80 \pm 0,87^{A}$ \\
\hline Lote A & $4,73 \pm 0,28^{A}$ & $16,47 \pm 0,71^{\mathrm{B}}$ \\
\hline Lote B & $2,65 \pm 0,26^{\mathrm{B}}$ & $8,53 \pm 0,19 c$ \\
\hline Lote C & $2,65 \pm 0,64^{B}$ & $8,42 \pm 1,35^{c}$ \\
\hline Lote D & $2,73 \pm 0,23^{B}$ & $8,54 \pm 0,39 c$ \\
\hline
\end{tabular}

A continuación, se compararon los valores de Km y Vmáx de los inhibidores y se observó que el Inhibidor I, no tuvo influencia sobre Vmáx, pero provocó un incremento en $\mathrm{Km}$ y por tanto se determinó el valor de Ki, como inhibidor competitivo. Además, el Inhibidor II generó una disminución en Vmáx y $\mathrm{Km}$, por lo cual se trató de un inhibidor mixto y se calcularon las constantes de inhibición Ki' para la unión del inhibidor con el complejo enzima sustrato y Ki de la unión entre la enzima y el inhibidor (Nelson y Cox, 2014). En la Tabla 9 se muestran los valores de Ki' y Ki para los dos tipos de inhibidores.

Tabla 9. Constantes de inhibición obtenidas para los inhibidores encontrados en el extracto de sangorache

\begin{tabular}{|c|c|c|}
\cline { 2 - 3 } \multicolumn{1}{c|}{} & Inhibidor I & Inhibidor II \\
\hline $\begin{array}{c}\text { Tipo de } \\
\text { inhibición }\end{array}$ & Competitiva & Mixta \\
\hline Ki' (mM) & - & 0,101 \\
\hline Ki (mM) & 0,066 & 0,274 \\
\hline
\end{tabular}

Al igual que la constante de Michaelis-Menten, que se considera como una medida del grado de afinidad entre la enzima y el sustrato, la constante de inhibición también brinda una idea de la relación existente entre la enzima y el inhibidor; de esta forma, mientras menor es su valor, mayor es su afinidad.

En la Tabla 9 se observa que la constante de inhibición (Ki) es menor en el Inhibidor 1, lo que sugiere mayor afinidad con la enzima; al contrario de lo que se muestra en el Inhibidor II, donde la Ki', que representa la interacción entre el inhibidor II y el complejo enzima-sustrato, es mayor a Ki, que simboliza la relación entre el mismo inhibidor con la enzima, por lo cual se podría decir que este inhibidor afecta de forma más significativa la disociación del complejo enzima-sustrato para la formación del producto (Voet y Voet, 2005). 


\section{Conclusiones y recomendaciones}

\subsection{Conclusiones}

Con base en el modelo matemático obtenido del análisis estadístico de las variables \%EI, \%AEI y $\%$ RAF, en la inmovilización de tripsina en sepharosa $6 \mathrm{~B}-\mathrm{CL}$, a $15^{\circ} \mathrm{C}$, se determinó que a un valor de $\mathrm{pH}$ de 10,5 y una carga de $25 \mathrm{mg} / \mathrm{mL}$ se alcanzarían valores máximos de 85,5; 98,1 y 29,27, respectivamente.

Los inhibidores de tripsina más activos fueron encontrados en el permeado de la semilla de sangorache con una AIE de 19,22 $\pm 0,4 \mathrm{mU} / \mathrm{mg}$ y en el retenido de la misma semilla con un valor de $39,88 \pm 0,69 \mathrm{mU} / \mathrm{mg}$.

La purificación por cromatografía de afinidad del permeado de la semilla de sangorache permitió obtener lotes con AIE de 1114,6 y 300,6 mU/mg; y, en el caso del retenido se obtuvieron lotes con AIE de 3288,3 y $461,1 \mathrm{mU} / \mathrm{mg}$.

La caracterización cinética de las fracciones inhibidoras principales mostró que existen dos tipos de inhibidores, uno competitivo (Ki: 0,066 mM) y otro mixto (Ki': 0,101 mM, Ki: 0,274 mM).

\subsection{Recomendaciones}

Se sugiere purificar por cromatografía de afinidad a los inhibidores presentes en las semillas de arveja, amaranto, chocho y fréjol, ya que también presentaron actividad inhibidora de tripsina.

Se recomienda estudiar el diseño de bioinsecticidas a partir de los inhibidores específicos obtenidos, ya que tendrían un mínimo impacto sobre otros organismos benéficos que se encuentren en el ecosistema.

\section{Bibliografía}

Aitken, A. y Laermonth, M. (2002). Protein determination by UV absorption. En Walker, J. (Ed.). Protocols Handbook (pp. 3-6). Totowa, Estados Unidos: Humana Press Inc

American Psychological Association. (2012). Publication manual of the American Psychological Association. Washington, DC: American Psychological Assoc.

Betancur, D., Guerrero, L., Hernández, V. y Marrufo, D. (2012). Inhibición de la Enzima Convertidora de Angiotensina I con hidrolizados proteicos de Jatropha curcas. Bioquímica clínica latinoamericana, 46(3), 385 - 393. Obtenido de http://www.scielo.org.ar/pdf/abcl/v46n3/v46n3a06.pdf .

Bonzón, E. (1996). Obtención de una Matriz de Afinidad de Tripsina-Glioxil-Sepharosa CL-4B para la Purificación del Inhibidor de Proteasas de Stichodactyla heliantus. (Trabajo de diploma). Universidad de la Habana, La Habana, Cuba. 
Castillo, P., Gómez, C., Sinche, M., Duchicela, J., \& Muñoz, R. (2012). Identificación de la actividad proteolítica en especies vegetales presentes en las provincias de Loja y Pastaza. Revista Politécnica, 31.

Chávez, M., González, Y., Hernández, A., Pascual, I., Reytor, M. y Rivero, M. (2011). Screening of protease inhibitory activity in extracts of five Ascidian species from Cuban coasts. Biotecnología Aplicada, 28(2), 77 - 82. Obtenido de http://scielo.sld.cu/pdf/bta/v28n2/bta 02211.pdf

Da Silva, A. M., Tavares, A. P., Rocha, C. M., Cristóvão, R. O., Teixeira, J. A., \& Macedo, E. A. (2012). Immobilization of commercial laccase on spent grain. Process biochemistry, 47(7), 1095-1101. Obtenido de http://www.sciencedirect.com/science/article/pii/S1359511312001 353

Del Monte, A., Cutiño, B., González, J., Chávez, M., Díaz, J. (2014). Diseño racional de la inmovilización de proteínas: aplicaciones en cromatografía de afinidad y bioconversión enzimática. Anales de la Academia de Ciencias de Cuba. Obtenido de http://www.revistaccuba.cu/index.php/acc/article/viewFile/187/145

Dos Santos, E., Oliveira, A., Rabêlo, L., Uchôa, A. y Araujo, A. (2012). Affinity Chromatography as a key tool purify protein protease inhibitors from plants. Obtenido de http://www.Intech open.com/books/affinity-chromatography/affinity-chromatography-asa-key-tool-to-purifyprotease-inhibitors-from-plants

Earlanger, B., Kokowski, N. y Cohen, W. (1961). The preparation and properties of two new chromogenic substrates of trypsin. Archives of Biochemistry and Biophysics, 95(2), 271 278.

Elizalde, A., Porilla, Y. y Chaparro, D. (2009). Factores antinutricionales en semillas. Facultad de Ciencias Agropecuarias, 7(1), 46. Obtenido de http://www.scielo.org.co/pdf/bsaa/v7n1/ v7n1a07.pdf

Freije, J., Mulder,P., Werkman, W., Rieux, L., Niederlander, H., Verpoorte, E., y Bischoff, R. (2005). Chemically modified, immobilized trypsin reactor with improved digestion efficiency. Journal of proteome research, 4(5), 1805-1813.

García, R., Salas, E., Del monte, A., Del Rivero, A., Guerra, Y. y Chávez M. (2009). Micro y nanobiotecnologías en la detección y caracterización de inhibidores de proteasas de interés biomédico. Revista Cubana de Física. 26(1). 76. Obtenido de http://www.fisica.uh.cu/ biblioteca/revcubfi/2009/vol.26-No.1/RCF-26-1-2009-76.pdf 
Habib, M. y Majid, K. (2007). Plant protease inhibitors: a defense strategy in plants. Biotechnology and Molecular Biology, 2(3), 68-85.

Koolman, J. y Röhm, K. (2004). Bioquímica texto y atlas. Madrid, España: Editorial Médida Panamericana.

Li, Y., Gao, F., Wei, W., Qu, J., Ma, G. y Zhou, W. (2010). Pore size of macroporous polystyrene microspheres affects lipase immobilization. Journal of Molecular Catalysis, 66 (1), 182-189.

Montgomery, D. (2003). Diseño y análisis de experimentos. México D.F., México: Limusa.

Müller-Esterl, F. (2008). Bioquímica. Fundamentos para medicina y ciencias de la vida. Obtenido de: http://books.google.com.ec/books?id=X2YV G6Fzp1UC\&printsec=frontcover\#v=one page $\& q \& f=f a l s e$

Nelson D. y Cox, M. (2014). Principles of biochemistry. Obtenido de https://archive.org/details/9788582710739

Ramírez, J., y Rangel, I. (2011). El frijol (Phaseolus vulgaris): su importancia nutricional y como fuente de fitoquímicos. Revista Fuente, 3(8).

Rocha, C., Gonçalves, P., y Teixeira, J. (2011). Immobilization of trypsin on spent grains for whey protein hydrolysis. Process biochemistry, 46(2), 505-511. Obtenido de http://www.sciencedirect.com/science/article/pii/S1359511310003843

Tripathi, G. (2009). Enzyme biotechnology. ABD Publishers. 